\title{
Rolling Balls and Octonions
}

\author{
A. A. Agrachev*
}

\begin{abstract}
In this semi-expository paper we disclose hidden symmetries of a classical nonholonomic kinematic model and try to explain geometric meaning of basic invariants of vector distributions.
\end{abstract}

\section{Introduction}

The paper is written to the 70th anniversary of Vladimir Igorevich Arnold. This is just a small mathematical souvenir but I hope that Vladimir Igorevich will get some pleasure looking over it. The content of the paper is well described by the cryptogram below. The figure represents the roots system of the exceptional group $G_{2}$ (the automorphisms group of octonions) and two touching each other circles whose diameters are in ratio 3:1.

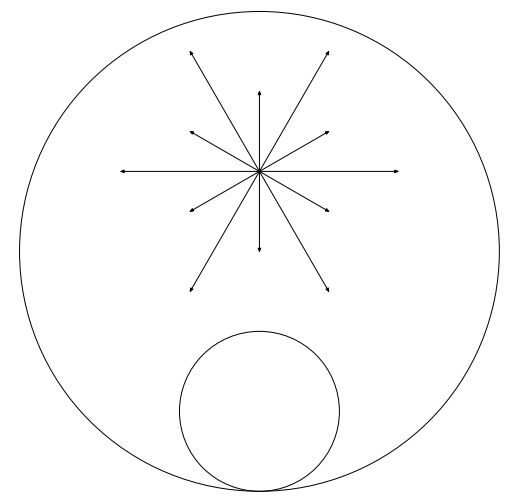

Our starting point is a classical nonholonomic kinematic system that is rather important in robotics: a rigid body rolling over a surface without

${ }^{*}$ SISSA-ISAS, Trieste \& Steklov Math. Inst., Moscow 
slipping or twisting. The surface is supposed to be the surface of another rigid body so that the situation is, in fact, symmetric: one body is rolling over another one. We also assume that the surfaces of the bodies are smooth and cannot touch each other in more than one point.

This system has a 5-dimensional configuration space: coordinates of the points on each surface where the surfaces touch each other give 4 parameters; fifth parameter measures mutual orientation of the bodies at the touching point.

Now let us imagine one of the bodies immovable and another rolling. Given an initial configuration, it is rather clear that one can roll the body in a unique way along any curve on the immovable surface starting from the initial touching point. In other words, given an initial configuration, admissible motions are parameterized by the curves on the two-dimensional surface with a fixed initial point. On the other hand, it is not hard to prove (see [2, Ch. 24]) that admissible motions allow to reach any configuration from any other as soon as two bodies are not congruent. We are thus in a typical nonholonomic situation since the configuration space is 5-dimensional.

Now turn to Mathematics. Admissible velocities (i.e. velocities of admissible motions) form a rank 2 vector distribution on the configuration space. This distribution is involutive if and only if our bodies are the balls of equal radii. More precisely, let vector fields $f, g$ form a local basis of our distribution, $[f, g]$ be the commutator (Lie bracket) of the fields, and $q$ a point of the configuration space. It happens that vectors $f(q), g(q),[f, g](q)$ are linearly independent if and only if the curvatures of two surfaces are not equal at their touching points corresponding to the configuration $q$. Moreover, if these curvatures are not equal, then:

$$
f(q) \wedge g(q) \wedge[f, g](q) \wedge[f,[f, g]](q) \wedge[g,[g, f]](q) \neq 0
$$

In other words, the basic fields, their first and second order Lie brackets are all linearly independent.

The germs of rank 2 distributions in $\mathbb{R}^{5}$ with property (1) were first studied by Elie Cartan in his famous paper [6]. A brief count of parameters demonstrates that 5 is the lowest dimension where the classification of generic germs of distributions must have functional invariants. This happens for rank 2 and rank 3 distributions in $\mathbb{R}^{5}$; moreover, the classifications for both values of the rank are essentially equivalent and it is sufficient to study the case of rank 2 . 
Cartan found a fundamental tensor invariant that is a degree 4 symmetric form on the distribution: the desired functional invariant is just the crossratio of the roots of this form. There is exactly one equivalence class for which the Cartan's form is identical zero. We call the germs from this class flat; a germ of the distribution is flat if and only if it admits a basis generating the 5-dimensional nilpotent Lie algebra. Cartan showed that symmetry group of a flat distribution is the split form of the 14-dimensional exceptional simple Lie group $G_{2}$. In all other cases, dimension of the symmetry group does not exceed 7 .

How all that is related to the rolling bodies? It is rather obvious that the group of symmetries of the "no slipping no twisting distribution" is trivial for generic bodies. This group acts transitively on the configuration space if and only if the surfaces of both bodies have constant curvatures. Let us stay with the case of constant nonnegative curvatures so that the surfaces are spheres (one of them may be a plane, i.e. the sphere of the infinite radius).

Natural symmetries are isometries of the spheres; they form a 6-dimensional Lie group. It was Robert Bryant who claimed first that no slipping no twisting distribution is flat in the case of spheres whose radii are in ratio 3:1. He insisted that just followed the Cartan's method and never published this fact in a paper or a preprint. It also remained absolutely unclear what are hidden additional symmetries (dimension of the symmetry group jumps from 6 to 14 when ratio of the radii gets $3: 1$ !). Unfortunately, Cartan's method does not give much in this regard: the construction of the fundamental invariant is based on the involved reduction-prolongation procedures in the jet spaces, and any connection with the original geometric problem is lost quite far from the end of the way.

The goal of this note is to finally untwine this puzzle. In Section 2 we give a simple twistor model for the configuration space of the rolling balls problem and for the no slipping no twisting distribution; the role of the group $G_{2}$ is not yet clear from this model.

Split form of $G_{2}$ is the group of automorphisms of split-octonions, a hyperbolic version of usual octonions, where positive definite quadratic form (square of the norm of octonions) is substituted by a non-degenerate signindefinite quadratic form. Split-octonions have nontrivial divisors of zero (zero locus of the sign-indefinite form). A simple quadratic transformation demonstrates that no slipping no twisting distribution is equivalent to the "divisors of zero distribution" of split-octonions if the radii of the balls are in ratio $3: 1$. This is the subject of Section 3 . 
In Section 4 we outline a recently developed variational way to construct differential invariants of vector distributions in order to put the rolling bodies model in a broader framework and to explain the meaning of basic invariants.

Acknowledgements. I am grateful to Robert Bryant, Aroldo Kaplan and Igor Zelenko for very interesting stimulating discussions.

\section{Twistor model}

We study admissible motions of two balls $B_{r}$ and $B_{R}$ rolling one over another without slipping or twisting. Here $r$ and $R$ are the radii of the balls. Instant configuration of the system of two balls is determined by an orientation preserving isometry of the tangent planes to the spheres $S_{r}=\partial B_{r}$ and $S_{R}=$ $\partial B_{R}$ at the points where the balls touch each other. In other words, the state space of our kinematic system is:

$$
M_{R, r}=\left\{\mu: T_{q_{1}} S_{r} \rightarrow T_{q_{2}} S_{R} \mid \begin{array}{c}
q_{1} \in S_{r}, q_{2} \in S_{R}, \mu \text { is an } \\
\text { isometry of oriented planes }
\end{array}\right\} .
$$

It is easy to see that $M_{R, r}$ is a smooth 5-dimensional manifold. Motions of the system are families of isometries $\mu(t): T_{q_{1}(t)} S_{r} \rightarrow T_{q_{2}(t)} S_{R}, t \in \mathbb{R}$. The no slipping condition reads:

$$
\mu(t)\left(\dot{q}_{1}(t)\right)=\dot{q}_{2}(t)
$$

The no twisting condition requires that $\mu(t)$ transforms parallel along $q_{1}(t)$ vector fields in the parallel along $q_{2}(t)$ vector fields.

These two conditions define a rank 2 vector distribution $D^{R, r}$ on $M_{R, r}$. We have: $D^{R, r}=\bigcup_{\mu \in M_{R, r}} D_{\mu}^{R, r}$, where $D_{\mu}^{R, r}$ is a two-dimensional subspace of $T_{\mu} M_{R, r}$; admissible motions of the two balls system are exactly integral curves of the distribution $D^{R, r}$. Given an initial configuration, the ball $B_{r}$ can be rolled in a unique way along any smooth curve on $S_{R}$ and the same is true if we transpose $r$ and $R$. In the formal geometric language this observation just means that the subspace $D_{\mu}^{R, r} \subset T_{\mu} M_{R, r}$, where $\mu: T_{q_{1}} \rightarrow T_{q_{2}}$, is projected one-to-one onto $T_{q_{1}} S_{R}$ and $T_{q_{2}} S_{r}, \forall \mu \in M_{R, r}$.

In what follows, we treat $S_{r}$ and $S_{R}$ as unit spheres in $\mathbb{R}^{3}$ with re-scaled metrics:

$$
M_{R, r}=\left\{\mu: q_{1}^{\perp} \rightarrow q_{2}^{\perp}\left|q_{i} \in \mathbb{R}^{3},\right| q_{i}|=1, i=1,2, R| \mu(v)|=r| v \mid, \forall v \in q_{1}^{\perp}\right\} .
$$


Let $\rho=\frac{R}{r}$, the homothety $\iota_{\rho}: \mu \mapsto \rho \mu, \mu \in M_{R, r}$, transforms $M_{R, r}$ in $M_{1,1}$. We set $D^{\rho}=\iota_{\rho *} D^{R, r}$. The distribution $D^{\rho}$ on $M_{1,1}$ is determined by the "re-scaled no slipping condition"

$$
\mu\left(\dot{q}_{1}(t)\right)=\rho \dot{q}_{2}(t)
$$

and the no twisting condition; the last one remains unchanged.

From now on we will deal with fixed space $M_{1,1}$ endowed with the family of distributions $D^{\rho}$ instead of the family of pairs $\left(M_{R, r}, D^{R, r}\right)$. In order to explicitly describe the distributions $D^{\rho}$ we use a classical quaternion's parameterization of spherical bundle $\mathfrak{p}: \mathcal{S} \rightarrow S^{2}$, to the unit sphere $S^{2} \subset \mathbb{R}^{3}$, where

$$
\mathcal{S}=\left\{(q, v) \in \mathbb{R}^{3} \times \mathbb{R}^{3}|| q|=| v \mid=1,\langle q, v\rangle=0\right\}, \quad \mathfrak{p}(q, v)=q .
$$

Let us recall this parameterization. We identify $\mathbb{R}^{3}$ with the space of imaginary quaternions:

$$
\mathbb{R}^{3}=\{\alpha i+\beta j+\gamma k \mid \alpha, \beta, \gamma \in \mathbb{R}\} \subset \mathbb{H} .
$$

Let $S^{3}=\{w \in \mathbb{H}|| w \mid=1\}$ be the group of unitary quaternions; then $\mathfrak{h}: S^{3} \rightarrow S^{2}, \mathfrak{h}(w)=\bar{w} i w$ is the classical Hopf bundle, while the mapping

$$
\Psi: S^{3} \rightarrow \mathcal{S}, \quad \Psi(w)=(\bar{w} i w, \bar{w} j w)
$$

is a double covering. Moreover, the diagram

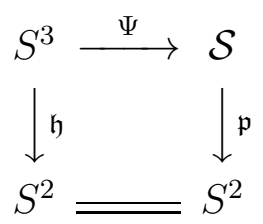

is commutative, hence $\Psi$ is a fiber-wise mapping of the bundle $\mathfrak{h}: S^{3} \stackrel{S^{1}}{\longrightarrow} S^{2}$ onto the bundle $\mathfrak{p}: \mathcal{S} \stackrel{S^{1}}{\longrightarrow} S^{2}$ which induces a double covering of the fibers. The fibers of the bundle $\mathfrak{h}: S^{3} \rightarrow S^{2}$ are the residue classes $\left\{e^{i \theta} w \mid \theta \in\right.$ $\mathbb{R} \bmod 2 \pi\}$ of the one-parametric subgroup generated by $i$.

The normal to the fibers distribution

$$
\operatorname{span}\{j w, k w\} \subset T_{w} S^{3}, \quad w \in S^{3}
$$


is a connection on the principal bundle $\mathfrak{h}: S^{3} \rightarrow S^{2}$. It is easy to see that $\Psi$ transforms this connection in the Levi Civita connection on the bundle $\mathfrak{p}: \mathcal{S} \rightarrow S^{2}$, which defines standard parallel transport on $S^{2}$.

We are now ready to give a quaternion's model of the rolling balls configuration space $M_{1,1}$ (more precisely, of a double covering to $M_{1,1}$ ) and of the no slipping no twisting distributions $D^{\rho}$. For any $w_{1}, w_{2} \in S^{3}$ there exists a unique orientation preserving isometry of the fiber

$$
\mathfrak{h}^{-1}\left(\mathfrak{h}\left(w_{1}\right)\right)=\left\{e^{i \theta} w_{1} \mid \theta \in \mathbb{R} \bmod 2 \pi\right\}
$$

on the fiber $\mathfrak{h}^{-1}\left(\mathfrak{h}\left(w_{2}\right)\right)$ that sends $w_{1}$ to $w_{2}$. This isometry sends $e^{i \theta} w_{1}$ to $e^{i \theta} w_{2}$. Moreover, pairs $\left(w_{1}, w_{2}\right)$ and $\left(w_{1}^{\prime}, w_{2}^{\prime}\right)$ define one and the same isometry if and only if $w_{1}^{\prime}=e^{i \theta^{\prime}} w_{1}, w_{2}^{\prime}=e^{i \theta^{\prime}} w_{1}$ (with one and the same $\theta^{\prime}$ ). Hence the coset space of $S^{3} \times S^{3}$ by the action $\left(w_{1}, w_{2}\right) \mapsto\left(e^{i \theta} w_{1}, e^{i \theta} w_{2}\right)$ of the one-parametric group $\left\{e^{i \theta} \mid \theta \in \mathbb{R} \bmod 2 \pi\right\}$ is a double covering of $M_{1,1}$. We use symbol $\mathbf{M}$ for this coset space and $\pi: S^{3} \times S^{3} \rightarrow \mathbf{M}$ for the canonical projection. By $\mathbf{D}^{\rho}=\bigcup_{\mathbf{x} \in \mathbf{D}} \mathbf{D}_{\mathbf{x}}^{\rho}$ we denote the rank 2 distribution on $\mathbf{M}$ that is the pullback of $D^{\rho}$ by the double covering $\mathbf{M} \rightarrow M_{1,1}$. Then

$$
\mathbf{D}_{\pi\left(w_{1}, w_{2}\right)}^{\rho}=\pi_{*} \operatorname{span}\left\{\left(j w_{1}, \rho j w_{2}\right),\left(k w_{1}, \rho k w_{2}\right)\right\} \quad \forall w_{1}, w_{2} \in S^{3} .
$$

Let us now treat the quaternionic space $\mathbb{H}^{2}=\left\{\left(w_{1}, w_{2}\right) \mid w_{i} \in \mathbb{H}\right\}$ as $\mathbb{C}^{4}$, where $w_{1}=z_{1}+z_{2} j, w_{2}=z_{3}+z_{4} j, z_{l} \in \mathbb{C}, l=1, \ldots, 4$. We see that $\mathbf{M}$ is nothing else but a complex projective conic,

$$
\mathbf{M}=\left\{z_{1}: z_{2}: z_{3}:\left.z_{4}|| z_{1}\right|^{2}+\left|z_{2}\right|^{2}=\left|z_{3}\right|^{2}+\left|z_{4}\right|^{2}\right\} \subset \mathbb{C P}^{3} .
$$

This conic is often called "the space of isotropic twistors". Moreover,

$$
\mathbf{D}_{\pi\left(w_{1}, w_{2}\right)}^{\rho}=\pi_{*} \mathbb{C} j\left(w_{1}, \rho w_{2}\right) .
$$

\section{Split-Octonions}

We also treat $\mathbb{H}^{2}$ as the algebra $\hat{\mathbb{O}}=\left\{w_{1}+\ell w_{2} \mid w_{i} \in \mathbb{H}\right\}$ of split-octonions, where

$$
(a+\ell b)(c+\ell d)=(a c+d \bar{b})+\ell(\bar{a} d+c b) .
$$


Let $x=w_{1}+\ell w_{2}, \bar{x}=\bar{w}_{1}-\ell \bar{w}_{2}$ and $Q(x)=\bar{x} x=\left|w_{1}\right|^{2}-\left|w_{2}\right|^{2}$. Then $Q(x y)=Q(x) Q(y)$ and $x^{-1}=\frac{\bar{x}}{Q(x)}$ as soon as $Q(x) \neq 0$; the cone $Q^{-1}(0)$ consists of the divisors of zero.

The automorphisms group of the algebra $\hat{\mathbb{O}}$ is the split-form of the exceptional Lie group $G_{2}$. These automorphisms preserve quadratic form $Q$ and hence its polarization $\mathbf{Q}(x, y)=\frac{1}{4}(Q(x+y)-Q(x-y))$. In particular, these automorphisms preserve the subspace $\mathbb{R}^{7}=\{x \in \hat{\mathbb{O}} \mid \mathbf{Q}(1, x)=0\}$ and the conic

$$
K=\left\{x \in \mathbb{R}^{7} \mid Q(x)=0\right\}=\{x \in \hat{\mathbb{O}} \mid x x=0\} .
$$

Moreover, the automorphisms group of $\hat{\mathbb{O}}$ acts transitively on the "spherization" $\mathbf{K}=\left\{\mathbb{R}_{+} x \mid x \in K \backslash 0\right\}=S^{2} \times S^{3}$ of the cone $K$.

To any $x \in K \backslash 0$ there associated a 3-dimensional subspace of divisors of 0

$$
\Delta_{x}=\left\{y \in \mathbb{R}^{7} \mid x y=0\right\} ;
$$

the "spherization" turns $\Delta_{x}$ into a 2-dimensional subspace $\Delta_{\mathbf{x}} \subset T_{\mathbf{x}} \mathbf{K}$, where $\mathbf{x}=\mathbb{R}_{+} x$. Obviously, the automorphisms group of $\hat{\mathbb{O}}$ preserves vector distribution $\boldsymbol{\Delta}=\left\{\boldsymbol{\Delta}_{\mathrm{x}}\right\}_{\mathbf{x} \in \mathbf{K}}$.

Proposition 1 The mapping $\Phi:\left(w_{1}+\ell w_{2}\right) \mapsto\left(w_{1}^{-1} i w_{1}+\ell\left(w_{1}^{-1} w_{2}\right)\right)$ induces the diffeomorphism of $\mathbf{M}$ onto $\mathbf{K}$. Moreover, the differential of this diffeomorphism transforms the "no slipping, no twisting" distribution $\mathbf{D}^{3}$ in the "divisors of zero" distribution $\boldsymbol{\Delta}$.

Proof. Let $\hat{\Phi}: \mathbf{M} \rightarrow \mathbf{K}$ be the mapping induced by $\Phi$. We give an explicit formula for $\hat{\Phi}^{-1}$ : take $v_{1} \in S^{2}, v_{1}=\mathfrak{h}\left(w_{1}\right)$, then

$$
\hat{\Phi}^{-1}\left(v_{1}+\ell v_{2}\right)=\pi\left(w_{1}+\ell\left(w_{1} v_{2}\right)\right), \quad \forall v_{2} \in S^{3} .
$$

Now we have to prove that $\Phi(x)\left(D_{x} \Phi y\right)=0$ for any $x=w_{1}+\ell w_{2}, y=$ $z j w_{1}+3 \ell\left(z j w_{2}\right)$ such that $\left|w_{1}\right|=\left|w_{2}\right|, z \in \mathbb{C}$. It is sufficient to make calculation in the case $\left|w_{1}\right|=\left|w_{2}\right|=1$. We have:

$$
D_{w_{1}+\ell w_{2}} \Phi\left(z j w_{1}+\ell\left(z j w_{2}\right)\right)=2 \bar{w}_{1} z k w_{1}+2 \ell\left(\bar{w}_{1} z j w_{2}\right) .
$$

The desired result now follows from the multiplication rule (2).

Let us give an explicit parameterization of the distribution $\boldsymbol{\Delta}$ on $\mathbf{K}$. First we parameterize $\mathbf{K}$ itself:

$$
\mathbf{K}=\left\{v_{1}+\ell v_{2}\left|v_{1} \in \mathbb{R}^{3}, v_{2} \in \mathbb{H},\right| v_{1}|=| v_{2} \mid=1\right\} .
$$


Then $\boldsymbol{\Delta}_{v_{1}+\ell v_{2}}=\left\{v_{1} u+\ell\left(u v_{2}\right) \mid u,\left(v_{1} u\right) \in \mathbb{R}^{3}\right\}$; this is a simple corollary of the multiplication rule (2). Let $v \in \mathbb{R}^{3},|v|=1$; the mapping $w \mapsto w+v w v$ maps $\mathbb{H}$ onto the subspace $\left\{u \mid u,(v u) \in \mathbb{R}^{3}\right\}$. Now we substitute $u$ by $w+v_{1} w v_{1}$ in the above description of $\boldsymbol{\Delta}_{v_{1}+\ell v_{2}}$ and obtain the final parameterization:

$$
\boldsymbol{\Delta}_{v_{1}+\ell v_{2}}=\left\{\left[v_{1}, w\right]+\ell\left(\left(w+v_{1} w v_{1}\right) v_{2}\right) \mid w \in \mathbb{H}\right\} .
$$

\section{Jacobi curves}

In this section we briefly describe the variational approach to differential invariants of vector distributions (see [1, 3, 14]) in order to put the rolling balls model in a wider perspective. This approach is based on the contemporary optimal control techniques and suggests an alternative to the classical equivalence method (see [6, 7, 10]) of Elie Cartan.

Rank $k$ vector distribution $\Delta$ on the $n$-dimensional smooth manifold $M$ is just a smooth vector subbundle of the tangent bundle $T M$ :

$$
\Delta=\bigcup_{q \in M} \Delta_{q}, \quad \Delta_{q} \subset T_{q} M, \quad \operatorname{dim} \Delta_{q}=k .
$$

Distributions $\Delta$ and $\Delta^{\prime}$ are called locally equivalent at $q_{0} \in M$ if there exists a neighborhood $O_{q_{0}} \subset M$ of $q_{0}$ and a diffeomorphism $\Phi: O_{q_{0}} \rightarrow O_{q_{0}}$ such that $\Phi_{*} \Delta_{q}=\Delta_{\Phi(q)}^{\prime}, \forall q \in O_{q_{0}}$.

A local basis of $\Delta$ is a $k$-tuple of smooth vector fields $f_{1}, \ldots, f_{k} \in \operatorname{Vec} M$ such that

$$
\Delta_{q}=\operatorname{span}\left\{f_{1}(q), \ldots, f_{k}(q)\right\}, q \in O_{q_{0}} .
$$

Given a local basis, one may compute the flag of the distribution:

$$
\Delta_{q}^{l}=\operatorname{span}\left\{\left(\operatorname{ad} f_{i_{j}} \cdots \operatorname{ad} f_{i_{1}} f_{i_{0}}\right)(q) \mid 0 \leq j<l\right\}, l=1,2, \ldots,
$$

where $\operatorname{ad} f g \stackrel{\text { def }}{=}[f, g]$ is the Lie bracket.

It is easy to see that the subspaces $\Delta_{q}^{l}$ do not depend on the local basis. We set $\Delta^{l}=\bigcup_{q \in M} \Delta_{q}^{l}$, a growing sequence of subsets in $T M$. This sequence stabilizes as soon as $\Delta^{l+1}=\Delta^{l}$. The distribution is involutive if and only if $\Delta^{2}=\Delta$, it is completely nonholonomic (our subject) if $\Delta^{l}=T M$ for sufficiently big $l$. Generic distributions are, of course, completely nonholonomic. 
Integral curves of the distribution are often called horizontal paths. It is convenient to consider all paths of class $H^{1}$, not only smooth ones. We thus have a Hilbert manifold $\Omega_{\Delta}$ of horizontal paths:

$$
\Omega_{\Delta}=\left\{\gamma \in H^{1}([0,1] ; M) \mid \dot{\gamma}(t) \in \Delta_{\gamma(t)} \text {, for a.e. } t \in[0,1]\right\} .
$$

Now consider boundary mappings

$$
\partial_{t}: \Omega_{\Delta} \rightarrow M \times M
$$

defined by the formula $\partial_{t}:(\gamma)=(\gamma(0), \gamma(t))$. It is easy to show that $\partial_{t}$ are smooth mappings.

Critical points of the mapping $\partial_{1}$ are called singular curves of $\Delta$. Any singular curve is automatically a critical point of $\partial_{t}, \forall t \in[0,1]$. Moreover, any singular curve possesses a singular extremal, i. e. an $H^{1}$-curve $\lambda:[0,1] \rightarrow$ $T^{*} M$ in the cotangent bundle to $M$ such that

$$
\lambda(t) \in T_{\gamma(t)}^{*} M \backslash\{0\}, \quad(\lambda(t),-\lambda(0)) D_{\gamma} \partial_{t}=0, \quad \forall t \in[0,1] .
$$

We set:

$$
\Delta_{q}^{\perp}=\left\{\nu \in T_{q}^{*} M \mid\left\langle\nu, \Delta_{q}\right\rangle=0, \nu \neq 0\right\}, \quad \Delta^{\perp}=\bigcup_{q \in M} \Delta_{q}^{\perp}
$$

Obviously, $\Delta^{\perp}$ is a smooth $(n+k)$-dimensional submanifold of $T^{*} M$ (the annihilator of $\Delta$ ).

Let $\sigma$ be the canonical symplectic structure on $T^{*} M$. Pontryagin Maximum Principle implies that a curve $\lambda$ in $T^{*} M$ is a singular extremal if and only if it is a characteristics of the form $\left.\sigma\right|_{\Delta^{\perp}}$; in other words,

$$
\lambda(t) \in \Delta^{\perp}, \quad \dot{\lambda}(t) \in \operatorname{ker}\left(\left.\sigma\right|_{\Delta^{\perp}}\right), \quad 0 \leq t \leq 1 .
$$

All singular extremals are contained in the characteristic variety

$$
C_{\Delta}=\left\{z \in \Delta^{\perp}\left|\operatorname{ker} \sigma_{z}\right|_{\Delta^{\perp}} \neq 0\right\} .
$$

We have: $C_{\Delta}=\Delta^{2 \perp}$ if $k=2 ; C_{\Delta}=\Delta^{\perp}$ if $k$ is odd; typically, $C_{\Delta}$ is a codim 1 submanifold of $\Delta^{\perp}$ if $k$ is even.

A complete description of singular extremals is a hard task; to simplify the job we focus only on the regular part of the characteristic variety. We set:

$$
C_{\Delta}^{0}=\left\{z \in C_{\Delta}\left|\operatorname{dim} \operatorname{ker} \sigma_{z}\right|_{\Delta^{\perp}} \leq 2,\left.\operatorname{dim} \operatorname{ker} \sigma_{z}\right|_{\Delta^{\perp}} \cap T_{z} C_{\Delta}=1\right\} .
$$




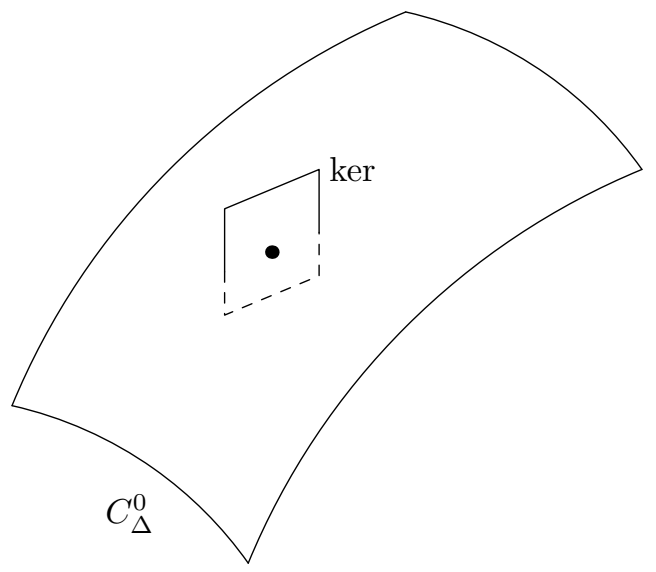

If $k=2$, then $C_{\Delta}^{0}=\Delta^{2 \perp} \backslash \Delta^{3 \perp}$.

$C_{\Delta}^{0}$ is a smooth submanifold of $\Delta^{\perp}$; it is foliated by singular extremals and by the fibers $T_{q}^{*} M \cap C_{\Delta}^{0}$.

The motion along singular extremals defines a local flow on $C_{\Delta}^{0}$; typically, this flow is not fiber-wise, i. e. it does not transform fibers into fibers (see the figure above).

Given $z \in C_{\Delta}^{0}$ and an appropriate small neighborhood $\mathcal{C}_{z}^{0}$ of $z$ in $C_{\Delta}^{0}$ we consider the canonical projection

$$
F: \mathcal{C}_{z}^{0} \longrightarrow \mathcal{C}_{z}^{0} /\{\text { singular extremals foliation }\}
$$

of $\mathcal{C}_{z}^{0}$ on the space of contained in $\mathcal{C}_{z}^{0}$ singular extremals.

Assume that $\lambda$ is a singular extremal through $z$ and it is associated to a singular curve $\gamma$, i. e. $\lambda(0)=z, \lambda(t) \in T_{\gamma(t)}^{*} M$. Consider a family of subspaces

$$
J_{\lambda}^{0}(t)=T_{\lambda} F\left(T_{\gamma(t)}^{*} M \cap \mathcal{C}_{z}^{0}\right)
$$

of the space

$$
T_{\lambda} \mathcal{C}_{z}^{0} /\{\text { singular extremals foliation }\} \cong T_{z} C_{\Delta}^{0} / T_{z} \lambda \text {. }
$$

Then $t \mapsto J_{\lambda}^{0}(t)$ is a smooth curve in the correspondent Grassmann manifold. Geometry of the curves $t \mapsto J_{\lambda}^{0}(\cdot)$ reflects the dynamics of the fibers along singular extremals and contains the fundamental information about distribution $\Delta$.

In what follows we assume that $k=2, n \geq 5$ and $\Delta_{q}^{2}, \Delta_{q}^{3}$ have maximal possible dimensions, i. e. $\operatorname{dim} \Delta_{q}^{2}=3, \operatorname{dim} \Delta_{q}^{3}=5$. 
i. First we consider the case $n=5$ that is the case studied by Cartan (see Introduction). Let $z \in T_{q}^{*} M$ and $\pi: T_{z}\left(T^{*} M\right) \rightarrow T_{q} M$ be the differential at $z$ of the projection $T^{*} M \rightarrow M$; then $\pi\left(J_{\lambda}^{0}(t)\right) \subset z^{\perp} \subset T_{q} M$. Moreover, $T_{q} \gamma \subset$ $\pi\left(J_{\lambda}^{0}(t)\right)$ and $t \mapsto \pi\left(J_{\lambda}^{0}(t)\right)$ is a curve in the projective plane $\mathbb{P}\left(z^{\perp} / T_{q} \gamma\right)$.

Proposition 2 (see [4]) A rank 2 distribution $\Delta$ on the 5-dimensional manifold is flat if and only if the curve $\pi\left(J_{\lambda}^{0}(\cdot)\right)$ is a quadric, for any singular extremal $\lambda$. .

In general, $\pi\left(J_{\lambda}^{0}(\cdot)\right)$ is not a quadric. Let $K_{z}(q) \subset z^{\perp}$ be the best approximating quadric for the curve $\pi\left(J_{\lambda}^{0}(\cdot)\right)$ near the point corresponding to the zero value of parameter $t$ (the osculating quadric of classical projective geometry); then $K_{z}(q)$ is zero locus of a signature $(2,1)$ quadratic form on $z^{\perp} / T_{q} \gamma$. We can, of course, treat $K_{z}(q)$ as zero locus of a degenerate quadratic form on $z^{\perp}$. Finally, $\mathcal{K}(q)=\bigcup_{z \in \Delta_{q}^{2 \perp}} K_{z}(q)$ is zero locus of a $(3,2)$ quadratic form on $T_{q} M$ (see [4] for details).

The family of quadratic cones $\mathcal{K}(q), q \in M$ is an intrinsically "raised" from $\Delta$ conformal structure on $M$ and $\Delta_{q} \subset K(q)$. This conformal structure was first found by Nurowski [11] who used the Cartan's equivalence method.

Remark. Nurowski's conformal structure has a particularly simple description for the divisors of zero distribution $\boldsymbol{\Delta}$ of Sec. 3. Namely, $\mathcal{K}(\mathbf{x})=$ $Q^{-1}(0) \cap T_{\mathbf{x}} \mathbf{K}, \mathbf{x} \in \mathbf{K}$, in this case.

ii. From now on $n$ is any integer greater or equal to 5 . Let $z \in C_{\Delta}^{0}, \lambda$ be the singular extremal through $z$ and $\gamma$ the correspondent singular curve. We set:

$$
J_{\lambda}(t)=D_{\lambda} F\left(\pi^{-1} \Delta_{\gamma(t)}\right) \subset T_{z} C_{\Delta}^{0} / T_{z} \lambda .
$$

Then $J_{\lambda}(t) \supset J_{\lambda}^{0}(t)$ and $J_{\lambda}(t)$ is a Lagrangian subspace of the symplectic space $T_{z} C_{\Delta}^{0} / T_{z} \lambda$. In other words, $J_{\lambda}(t)^{<}=J_{\lambda}(t)$, where

$$
\mathcal{S}^{<} \stackrel{\text { def }}{=}\left\{\zeta \in T_{z} C_{\Delta}^{0}: \sigma(\zeta, \mathcal{S})=0\right\}, \mathcal{S} \subset T_{z}
$$

Given $s \in \mathbb{R} \backslash\{0\}, s \lambda$ is the singular extremal through $s z \in C_{\Delta}^{0}$. Hence $T_{z}(\mathbb{R} z) \subset J_{\lambda}(t), \forall t$ and $J_{\lambda}(t) \subset T_{z}(\mathbb{R} z)^{<}$. This inclusion allows to make one more useful reduction. Namely, we set $\Sigma_{z}=T_{z}(\mathbb{R} z)^{<} / T_{z} \mathbb{R} z$, a symplectic space, $\operatorname{dim} \Sigma_{z}=2(n-3)$ and $J_{\lambda}(t)$ is a Lagrangian subspace of $\Sigma_{z}$. 
Let $L\left(\Sigma_{z}\right)$ be the Lagrange Grassmannian: the manifold formed by all Lagrangian subspaces of $\Sigma_{z}$. The curve $t \mapsto J_{\lambda}(t)$ considered as a curve in $L\left(\Sigma_{z}\right)$ is called the Jacobi curve associated to the extremal $\lambda$.

The dimension of a Lagrangian subspace is one half of the dimension of the ambient symplectic space. In particular, generic pair of Lagrangian subspaces has zero intersection. Jacobi curves are not at all generic, nevertheless, under very mild regularity assumption on the distribution (see [14]) they satisfy the following important property: $J_{\lambda}(t) \cap J_{\lambda}(\tau)=0$ for sufficiently small $|t-\tau| \neq 0$.

Let $\pi_{t \tau}$ be the linear projector of $\Sigma_{z}$ on $J_{\lambda}(\tau)$ along $J_{\lambda}(t)$. In other words, $\pi_{t \tau}: \Sigma_{z} \rightarrow \Sigma_{z}$

$$
\left.\pi_{t \tau}\right|_{J_{\lambda}(t)}=0,\left.\quad \pi_{t \tau}\right|_{J_{\lambda}(\tau)}=\mathbf{1}
$$

Lemma 1 (see[3]) We have:

$$
\operatorname{tr}\left(\frac{\partial^{2} \pi_{t \tau}}{\partial t \partial \tau}\right)=\frac{(n-3)^{2}}{(t-\tau)^{2}}+g_{\lambda}(t, \tau),
$$

where $g_{\lambda}(t, \tau)$ is a symmetric function of $(t, \tau)$ that is smooth in a neighborhood of $(t, t)$, for all $t$ out of a discrete subset of the domain of $J_{\lambda}(\cdot)$.

In what follows, we tacitly assume that the value of $t$ is taken out of the discrete subset provided by the lemma. A basic invariant of the parameterized singular extremal $\lambda$ is the generalized Ricci curvature

$$
\mathfrak{r}_{\lambda}(\lambda(t)) \stackrel{\text { def }}{=} g_{\lambda}(t, t) .
$$

Generalized Ricci curvature depends on the parameterization of the extremal; this dependence is controlled by the following chain rule. Let $\varphi: \mathbb{R} \rightarrow \mathbb{R}$ be a change of the parameter, then:

$$
\mathfrak{r}_{\lambda \circ \varphi}(\lambda(\varphi(t)))=\mathfrak{r}_{\lambda}(\lambda(\varphi(t))) \dot{\varphi}^{2}(t)+(n-3)^{2} \mathbb{S}(\varphi),
$$

where $\mathbb{S}(\varphi)=\frac{\dddot{\varphi}(t)}{2 \dot{\varphi}(t)}-\frac{3}{4}\left(\frac{\ddot{\varphi}(t)}{\dot{\varphi}(t)}\right)^{2}$, the Schwartzian derivative. The chain rule implies that the generalized Ricci curvature $\mathfrak{r}_{\lambda}$ can be always made zero by a local reparameterization of the extremal $\lambda$. We say that a local parameter $t$ is a projective parameter if $\mathfrak{r}_{\lambda}(t) \equiv 0$; such a parameter is defined up to a Möbius transformation. 
Let $t$ be a projective parameter, then the quantity:

$$
A(\lambda(t))=\left.\frac{\partial^{2} g}{\partial \tau^{2}}(t, \tau)\right|_{\tau=t}(d t)^{4}
$$

is a well defined degree 4 differential on $\lambda$; we called it the fundamental form on $\lambda$.

In arbitrary, not necessary projective parameter, the fundamental form has the following expression:

$$
A(\lambda(t))=\left(\left.\frac{\partial^{2} g}{\partial \tau^{2}}\right|_{\tau=t}-\frac{3}{5(n-3)^{2}} \mathfrak{r}_{\lambda}(t)^{2}-\frac{3}{2} \ddot{\mathfrak{r}}_{\lambda}(t)\right)(d t)^{4}
$$

Assume that $A(\lambda(t)) \neq 0$, then the identity $\left|A(\lambda(s))\left(\frac{d}{d s}\right)\right|=1$ defines a unique (up to a translation) normal parameter $s$.

Let $z \in C_{\Delta}^{0}$ and $\lambda_{s}$ be the normally parameterized singular extremal through $z$. We set

$$
\overline{\mathfrak{r}}(z)=\mathfrak{r}_{\lambda_{s}}(z),
$$

the projective generalized Ricci curvature. Then $z \mapsto \overline{\mathfrak{r}}(z)$ is a function on $C_{\Delta}^{0}$ which depends only on $\Delta$.

Now come back to the case $k=2, n=5$. In this case, the fundamental form $A$ is reduced to the famous Cartan's degree four form on the distribution constructed in [6] by the method of equivalence. The distribution is flat if and only if $A \equiv 0$.

Zelenko [14 performed detailed calculations for the rolling balls model. As before, let $\rho$ be the ratio of the radii of the balls. We assume that $1<\rho \leq+\infty$. It appears that

$$
\operatorname{sign}(A)=\operatorname{sign}(\rho-3)
$$

Singular curves are just rolling motions along geodesics (i.e. along big circles). The symmetry group acts transitively on the space of geodesics, hence function $\mathfrak{r}$ must be constant in this case. We have:

$$
\overline{\mathfrak{r}}=\frac{4 \sqrt{35}\left(\rho^{2}+1\right)}{3 \sqrt{\left(\rho^{2}-9\right)\left(9 \rho^{2}-1\right)}} .
$$

In particular, the distributions corresponding to different $\rho$ are mutually non equivalent and only the distribution corresponding to $\rho=3$ is flat. 


\section{References}

[1] A. Agrachev, Feedback-invariant optimal control theory and differential geometry, II. Jacobi curves for singular extremals. J. Dynamical and Control Systems, 1998, v.4, 583-604

[2] A. Agrachev, Yu. Sachkov, Control theory from the geometric viewpoint. Springer Verlag, 2004, xiv+412 pp.

[3] A. Agrachev, I. Zelenko, Geometry of Jacobi curves, I, II. J. Dynamical and Control Systems, 2002, v.8, 93-140; 167-215

[4] A. Agrachev, I. Zelenko, Nurovski's conformal structure for (2,5)distributions via dynamics of abnormal extremals. Proceed. RIMS workshop" Developments of Cartan geometry and related mathematical problems"; Kyoto, 2006, 204-218

[5] R. Bryant, L. Hsu, Rigidity of integral curves of rank 2 distributions. Invent. Math., 1993, v.114, 435-461

[6] E. Cartan, Les systèmes de Pfaff à cinq variables et les équations aux dérivées partielles du second ordre. Ann Sci. Ecole Normale, 1910, v.27(3), 109-192

[7] R. Gardner, The method of equivalence and its applications. SIAM, 1989 viii+127 pp.

[8] V. Jurdjevic, Geometric control theory. Cambridge Univ. Press, 1997, xviii+492 pp.

[9] A. Kaplan, F. Levstein, A split Fano plane. In preparation

[10] R. Mongomery, A tour of subRiemannian geometries, their geodesics and applications. Amer. Math Soc., 2002, xx+259 pp.

[11] P. Nurowski, Differential equations and conformal structures. J. Geometry and Physics, 2005, v.55, 19-49

[12] K. Sagerschnig, Split octonions and generic rank 2 distributions in dimension 5. Proceed. 26th Winter School on Geometry and Physics, Srni 2006" Rend. Crc. Mat. Palermo Suppl. II, to appear 
[13] T. Springer, F. Veldkamp, Octonians, Jordan algebras and exceptional groups. Springer Verlag, 2000, viii+208 pp.

[14] I. Zelenko, Variational approach to differential invariants of rank 2 vector distributions. J. Differential Geometry and Appl., 2006, v.24, 235259 